(Lomnytska et al., 2018). Expression of P-selectin positive platelet microvesicles was 2-fold higher in ovarian cancer $(\mathrm{p}<0.05)$. Increased expression of our identified markers on P-selectin positive platelet microvesicles was observed in ovarian cancer (figure 1).

Conclusion* Identified platelet biomarkers (Lomnytska et al. 2018) are detectable on platelet microvesicles in blood plasma with increased expression in ovarian cancer. Further analysis of microvesicles in relation to ultrasound evaluation has the potential to improve ovarian cancer diagnostics.

\section{ULTRASTAGING PROTOCOL IN SENTINEL LYMPH NODE FOR APPARENT EARLY STAGE OVARIAN CANCER}

V Lago*, B Montero, S Lopez, P Padilla Iserte, MT Luis Javier, B Segarra Vidal, S Domingo. University Hospital La Fe, Spain

\subsection{6/ijgc-2021-ESG0.407}

Introduction/Background* The objective of the present study is to determine the role of sentinel lymph node (SLN) ultrastaging in apparent early-stage ovarian cancer.

Methodology We previously demonstrated the feasibility of SLN in early-stage ovarian cancer in a pilot study and in a clinical trial (NCT03452982). The SLN of the 30 patients involved in both were processed following an ultrastaging protocol. The cost of ultrastaging processing was also reported.

Result(s)* A SLN was detected in up to $91.3 \%$ and $90 \%$ in the pelvic and para-aortic region, respectively. In all cases, a SLN was detected at least in one field, pelvic or para-aortic. The mean time from injection to SLN resection was $53.3 \pm$ $20.3 \mathrm{~min}$. Two of $30(6.6 \%)$ patients had a contralateral SLN in the para-aortic field, but no patients had contralateral SLN within the pelvic field after injection. The mean number of harvested SLN was $2.1 \pm 1.4$ (range: 0-5) and $2.7 \pm 1.5$ (range: $0-7$ ) in the pelvic and para-aortic region, respectively. Two patients were upgraded to stage IIIA1 because of lymph node metastasis. In the first case, based on single sections and haematoxylin and eosin (H\&E) examination, a pelvic
SLN micrometastasis $(1 \mathrm{~mm})$ was found on the first $\mathrm{H} \& \mathrm{E}$ section. By using the ultrastaging protocol, the size of the metastasis was increased to $2.1 \mathrm{~mm}$ (macrometastasis). In the same patient, the ultrastaging study of the inframesenteric para-cava SLNs found isolated tumour cells in the subcapsular and interfollicular lymph nodes sinus in one of the two SLN harvested (in one of the sections at the fourth and fifth ultrastage levels). The other upstaged case was a para-aortic macrometastasis in a patient in whom the SLN was not identified in the para-aortic field because of the absence of migration from the infundibulo-pelvic stump injection. The cost of ultrastaging in on average $96.8 €$ (range: 0-230.5) and $124.5 €$ (range: 0-322.7€) for pelvic and para-aortic SLN, respectively.

Conclusion* A uniform protocol for ultrastaging is essential for lower-volume metastasis detection and to provide reproducible information between upcoming studies, as evidence about SLN in ovarian cancer is growing.

\section{Ovarian cancer sentinel lymph node ultrastaging protocol}

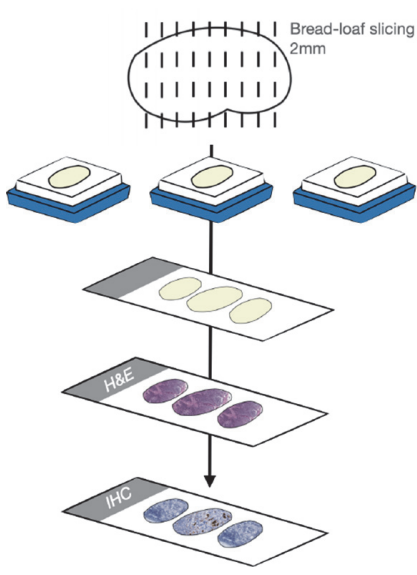

Macroscopic study

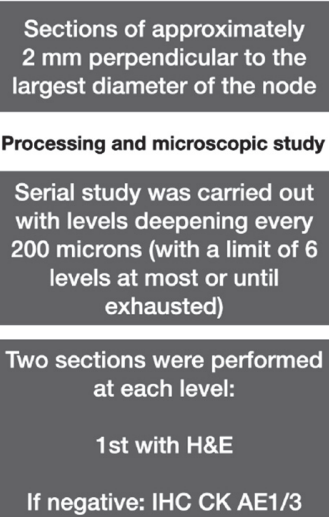

Abstract 468 Figure 2
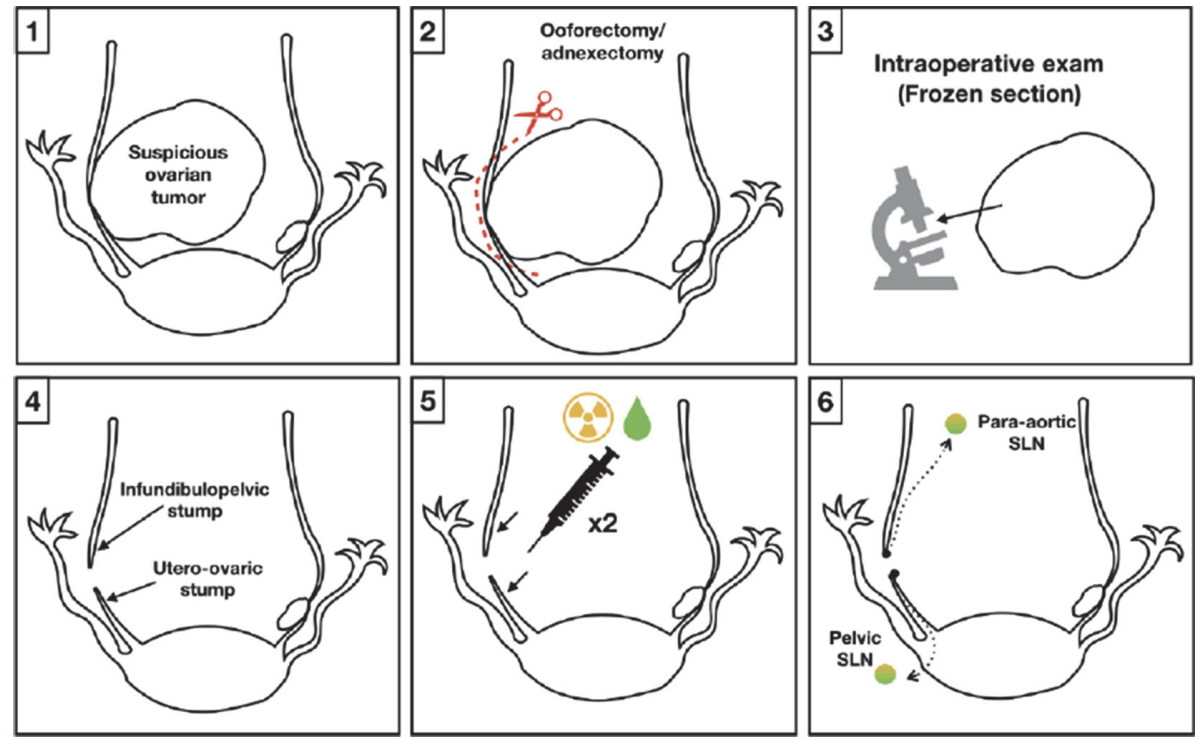

Abstract 468 Figure 1 